\title{
Depth first search in claw-free graphs
}

\author{
EXTENDED ABSTRACT
}

\author{
Gábor Wiener* \\ Department of Computer Science and Information Theory \\ Budapest University of Technology and Economics \\ e-mail: wiener@cs.bme.hu
}

All graphs in this paper are simple, finite, and undirected; the vertex set of a graph $G$ is denoted by $V(G)$. A graph is claw-free if it does not contain $K_{1,3}$ as an induced subgraph. A graph $G$ is traceable if it contains a hamiltonian path. The minimum leaf number $\operatorname{ml}(G)$ is the minimum number of leaves (vertices of degree 1) of the spanning trees of $G$. The minimum branch number $\mathrm{s}(G)$ is the minimum number of branches (vertices of degree at least 3) of the spanning trees of $G$. A tree $T$ is a $k$-tree if all vertices have degree at most $k$. The minimum degree of $G$ is denoted by $\delta(G)$ and the minimum sum of degrees of $k$ independent vertices of $G$ is denoted by $\delta_{k}(G)$. The depth first search (DFS) of a connected graph $G$ (see e.g. [4]) produces a spanning tree of $G$, called a DFS-tree, rooted at some node $r$; the leaves of a DFS-tree different from $r$ will be called $d$-leaves of the DFS-tree.

Hamiltonian properties of claw-free graphs have been examined for more than three decades; one of the early results is due to Matthews and Sumner [6] and was also found independently by Liu, Tian, and Wu [5].

Theorem 1. (Matthews and Sumner, Liu et al., 1985) Let $G$ be a connected clawfree graph of order $n$. If $\delta_{3}(G) \geq n-2$, then $G$ is traceable.

Gargano, Hammar, Hell, Stacho, and Vaccaro [2] proved a generalization of Theorem 1 concerning the minimum branch number.

Theorem 2. (Gargano et al., 2002) Let $G$ be a connected claw-free graph of order $n$ and let $k$ be a nonnegative integer. If $\delta_{k+3}(G) \geq n-k-2$, then $\mathrm{s}(G) \leq k$.

This result was generalized further by Salamon [7].

Theorem 3. (Salamon, 2010) Let $G$ be a connected claw-free graph of order $n$ and let $k$ be a nonnegative integer. If $\delta_{k+1}(G) \geq n-k$, then $\operatorname{ml}(G) \leq k$.

Since a branch vertex has degree at least 3 , it is obvious that $\operatorname{ml}(G) \geq \mathrm{s}(G)+2$, thus Theorem 3 is a generalization of Theorem 2 indeed. Theorem 3 was rediscovered in 2012 by Kano, Kyaw, Matsuda, Ozeki, Saito, and Yamashita [3] and they also proved a stronger version.

Theorem 4. (Kano et al., 2012) Let $G$ be a connected claw-free graph of order $n$ and let $k$ be a nonnegative integer. If $\delta_{k+1}(G) \geq n-k$, then $G$ has a spanning 3-tree with at most $k$ leaves.

The main result of the paper is the following theorem.

\footnotetext{
*Research was supported by the National Research, Development and Innovation Office - NKFIH, grant no. OTKA 108947 and by the János Bolyai Research Scholarship of the Hungarian Academy of Sciences.
} 
Theorem 5. Every connected claw-free graph G has a DFS-tree T such that no two of the d-leaves of $T$ have a common neighbour. Moreover, if $v$ is not a cut vertex of $G$, then $T$ can be chosen such that it is rooted at $v$.

Though the proof of Theorem 5 is really short, it is omitted here due to the page limit. On the other hand, we sketch how Theorem 5 implies Theorem 4 . Let $G$ be a connected claw-free graph of order $n$ with $\delta_{k+1}(G) \geq n-k$ and let $T$ be a DFS-tree guaranteed by Theorem 5 . The set of d-leaves $D$ of $T$ is obviously an independent set, thus the degree sum of the vertices of $D$ is at most $n-|D|$, since all vertices in $V(G)-D$ has at most one neighbour in $D$. Hence $|D| \leq k$, that is $T$ has at most $k+1$ leaves. Notice that $T$, like any DFS-tree of a claw-free graph is a 3 -tree. In order to find a spanning 3-tree with at most $k$ leaves, we need a further local improvement step, which is omitted here due to lack of space.

Theorem 5 has some other connections with results concerning claw-free graphs, of which we only mention the following corollary.

Corollary 6. Let $G$ be a connected claw-free graph of diameter at most 2 and let $v$ be a non-cut vertex of $G$. Then there exists a hamiltonian path of $G$ starting at $v$.

Proof. By Theorem 5, there exists a DFS-tree $T$ of $G$ rooted at $v$, such that no two of the d-leaves of $T$ have a common neighbour. Since the diameter of $G$ is at most 2 , this is possible only if $T$ has just one d-leaf, which finishes the proof.

Corollary 6 is a stronger form of a result of Ainouche, Broersma, and Veldman [1] stating that every connected claw-free graph of diameter at most 2 is traceable (actually they also proved the more general theorem that all $m$-connected claw-free graphs $G$ with $\alpha\left(G^{2}\right) \leq m+1$ are traceable).

\section{References}

[1] A. Ainouche, H.J. Broersma, and H.J. Veldman, Remarks on hamiltonian properties of claw-free graphs, Ars. Combin. 29C (1990), 110-121.

[2] L. Gargano, M. Hammar, P. Hell, L. Stacho, and U. Vaccaro, Spanning spiders and light-splitting switches, Discrete Mathematics 285 (2004), 83-95. (Earlier versions: L. Gargano, P. Hell, L. Stacho, U. Vaccaro, Spanning trees with bounded number of branch vertices, ICALP02, Lecture Notes in Computer Science 2380 (2002), 355-365. and L. Gargano, M. Hammar, There are spanning spiders in dense graphs (and we know how to find them), ICALP03, Lecture Notes in Computer Science 2719 (2003), 802-816.)

[3] M. Kano, A. Kyaw, H. Matsuda, K. Ozeki, A. Saito, and T. Yamashita, Spanning trees with small number of leaves in a claw-free graph, Ars Combin. 103 (2012), 137-154.

[4] J. van Leeuwen (ed.), Handbook of Theoretical Computer Science A: Algorithms and Complexity, Elsevier, 1990.

[5] Y. Liu, F. Tian, and Z. Wu, Some results on longest paths and cycles in $K_{1,3}$-free graphs, J. Changsha Railway Inst. 4 (1986), 105-106.

[6] M.M. Matthews and D.P. Sumner, Longest paths and cycles in $K_{1,3}$-free graphs, J. Graph Theory 9 (2) (1985), 269-277.

[7] G. Salamon, Degree-Based Spanning Tree Optimization, PhD Thesis, Budapest University of Technology and Economics, http://doktori.math.bme. $\mathrm{hu} /$ Ertekezesek/salamon_dissertation.pdf (2010) 